Research, Society and Development, v. 9, n. 10, e2539108637, 2020

(CC BY 4.0) | ISSN 2525-3409 | DOI: http://dx.doi.org/10.33448/rsd-v9i10.8637

\title{
Regionalização da curva de regularização de vazão da bacia do rio Juruena
}

Regionalization of the streamflow regularization curve of the Juruena basin

Regionalización de la curva de regularización de flujos de la cuenca Juruena

Recebido: 21/09/2020 | Revisado: 22/09/2020 | Aceito: 23/09/2020 | Publicado: 25/09/2020

João Paulo de Amorim Oliveira

ORCID: https://orcid.org/0000-0002-5810-4705

Universidade Federal de Mato Grosso, Brasil

E-mail: jp_olyveira98@outlook.com

Eduardo Morgan Uliana

ORCID: https://orcid.org/0000-0003-2107-4634

Universidade Federal de Mato Grosso, Brasil

E-mail: morganuliana@ufmt.br

Múcio André dos Santos Alves Mendes

ORCID: https://orcid.org/0000-0002-8051-7805

Universidade Federal de Mato Grosso, Brasil

E-mail: mucioandre@gmail.com

Luana Lisboa

ORCID: https://orcid.org/0000-0002-7902-6072

Companhia de Pesquisa de Recursos Minerais, Brasil

E-mail: luana.lisboa@cprm.gov.br

\section{Resumo}

A regularização de vazão é essencial quando a demanda de água é maior que a vazão mínima. Isso torna necessária a acumulação dos excessos para atender períodos cuja disponibilidade hídrica natural é menor que demanda de captação. Nesse contexto, o objetivo do trabalho foi regionalizar a curva de regularização de vazões para a bacia do rio Juruena de forma a subsidiar projetos de outorga e dimensionamento de reservatórios. Foi gerada curva adimensional de regularização de vazão para sete seções de monitoramento de vazão da bacia. Em seguida foi ajustado o modelo potencial aos dados das curvas de forma a regionaliza-las. As equações de regionalização propostas possuem precisão e acurácia e poderão embasar projetos de regularização de vazão para irrigação, abastecimento público e geração de energia elétrica. 
Palavras-chave: Vazão; Reservatórios; Amazônia; Planejamento de recursos hídricos.

\begin{abstract}
Streamflow regularization is essential when the water demand is greater than the minimum streamflow. This makes it necessary to accumulate excesses to meet periods in which natural water availability is less than the demand for abstraction. In this context, the objective of the work was to regionalize the streamflow regularization curve for the Juruena river basin in order to subsidize projects for granting and dimensioning reservoirs. A dimensionless streamflow smoothing curve was generated for seven stream gaging station. Then the potential model was adjusted to the data of the curves in order to regionalize them. The proposed regionalization equations have precision and accuracy and can be used as a basis for streamflow regulation projects for irrigation, public supply and electricity generation.
\end{abstract}

Keywords: Streamflow; Reservoirs; Amazon; Water resources planning.

\title{
Resumen
}

La regularización de caudal es fundamental cuando la demanda de agua es mayor que la oferta mínima, siendo necesaria la acumulación de excesos para atender periodos críticos. En este contexto, el objetivo del trabajo fue regionalizar la curva de regularización de caudales de la cuenca del río Juruena, procurando subsidiar proyectos de captación de agua y dimensionamiento de embalses. Se generó una curva de suavizado de flujo adimensional para siete secciones de monitoreo fluviometricas en la cuenca. Luego, el modelo potencial obtuvo el mejor ajuste a los datos de las curvas para ser regionalizarlas. Las ecuaciones de regionalización propuestas poseen precisión y exactitud y pueden utilizarse como base para proyectos de regulación de caudales para riego, suministro público y generación eléctrica.

Palabras clave: Caudal, Embalses, Amazonia, Planificación de recursos hídricos.

\section{Introdução}

O estudo das vazões em cursos de água embasa o planejamento e a gestão de recursos hídricos superficiais. Destacam-se as vazões máxima, mínima e média as quais são obtidas a partir de análises estatísticas da série histórica registrada em estações fluviométricas (Pruski, Silva \& Koetz, 2006; Lopes et al., 2016).

O dimensionamento de reservatórios para regularização de vazões requer o conhecimento dessas três vazões. Segundo Collischon \& Dorneles (2015) e Uliana et al. 
(2016), nesse tipo de obra a vazão máxima é utilizada no dimensionamento estrutural e do vertedor do barramento. A descarga de fundo deve ser calculada com referência na vazão mínima e em normas específicas de outorga. Por fim, destaca-se a aplicação da vazão média de longa duração, utilizada junto com a mínima para calcular o volume do reservatório para atender determinada demanda.

A regularização de vazão é essencial quando a demanda de água é maior que a vazão mínima. Isso torna necessária a acumulação dos excessos para atender períodos cuja disponibilidade hídrica natural é menor que demanda de captação (Tucci \& Clarke, 2016).

O cálculo incorreto do volume do reservatório pode acarretar em falhas do mesmo para atender a demanda de água do empreendimento no período de estiagem. Uma das formas de se determinar o volume do reservatório é por meio de simulação da diferença entre as vazões naturais afluentes ao reservatório e a vazão demandada. Essa simulação é realizada diariamente para toda a série histórica, e assim, determina-se os déficits de vazão. O volume do reservatório é dimensionado considerando o pior cenário, neste caso o maior déficit entre a vazão afluente e a vazão captada no curso de água (Pruski, Silva \& Koetz, 2006; Tucci \& Clarke, 2016).

Para a análise explicitada calcula-se o volume do reservatório para atender diferentes demandas em relação a vazão média de longa duração. Enfatiza-se que o máximo de água passível de regularização/retirada no curso de água é 100\% da vazão média de longa duração (Qmld). Plotando-se a demanda utilizada em relação a Qmld e o volume do reservatório para atender essa demanda obtêm-se assim a curva de regularização de vazões.

A curva de regularização de vazão pode ser obtida apenas para locais com medição de vazão e séries históricas estabelecidas. Para obtenção da curva em locais sem medição devese realizar a regionalização da mesma de forma que o projetista consiga estimar o volume do reservatório em determinado ponto da bacia (Tucci, 2002).

A bacia do rio Juruena, localizada no Estado de Mato Grosso possui poucas estações de medição de vazão e possui demanda, em alguns cursos de água, da construção de reservatórios para irrigação e geração de energia elétrica.

Nesse contexto, o objetivo do trabalho foi regionalizar a curva de regularização de vazões para a bacia do rio Juruena de forma a subsidiar projetos de outorga e dimensionamento de reservatórios. 


\section{Metodologia}

\section{Área de estudo e base de dados}

O estudo foi realizado na bacia hidrográfica do rio Juruena (Figura 1), Estado de Mato Grosso. O rio Juruena ao encontrar o rio Teles Pires forma o rio Tapajós, afluente importante da margem direita do rio Amazonas. Na Figura 1 pode ser observada a localização da bacia do rio Juruena bem como a localização das estações fluviométricas utilizadas no estudo com as respectivas áreas de drenagem e hipsometria.

Figura 1. Mapa da bacia do rio Juruena, localização das estações fluviométricas e hipsometria das sub-bacias.

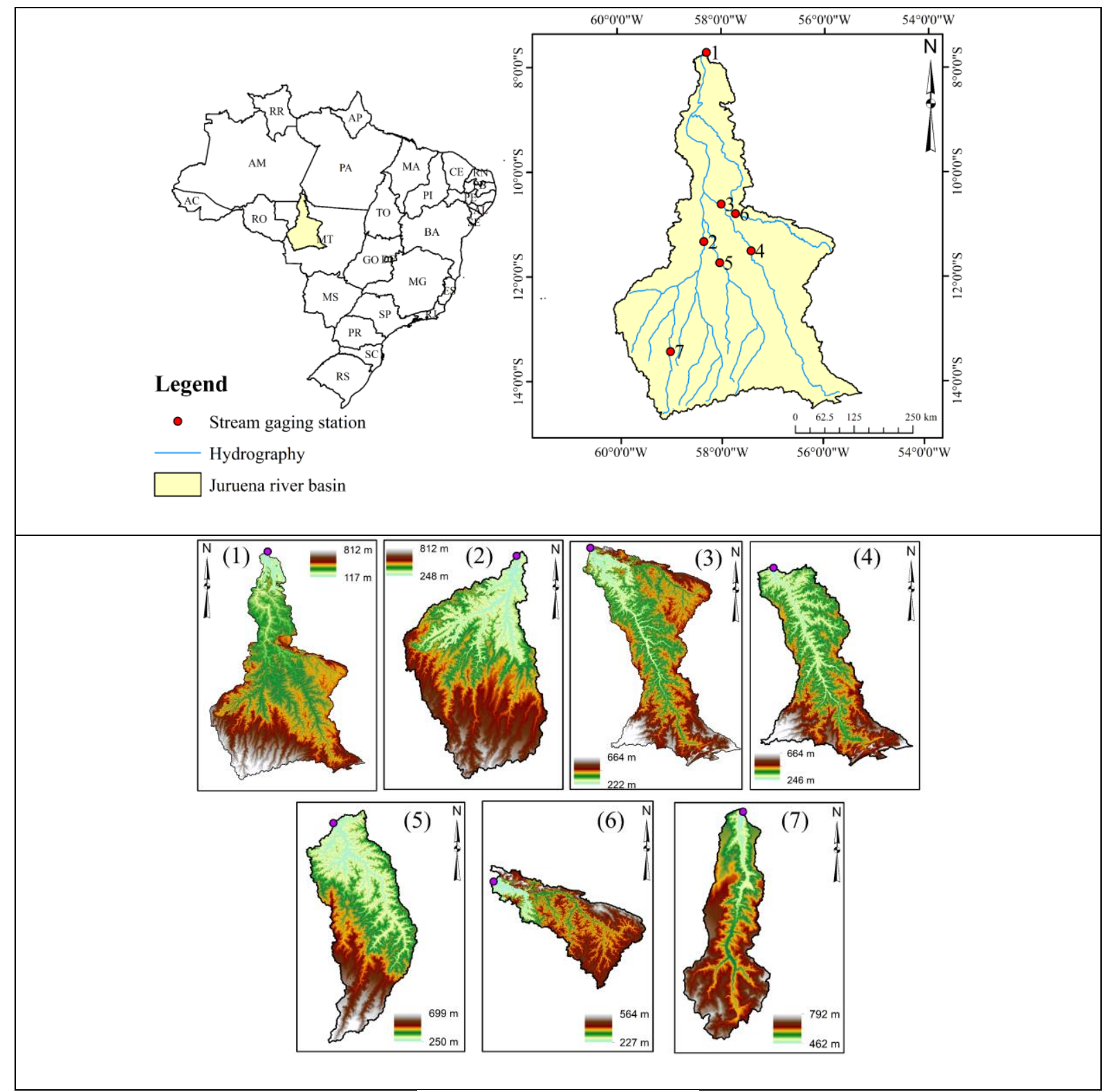

Fonte: Matos et al. (2020). 
$\mathrm{Na}$ Tabela 1 estão apresentados dados relacionados as estações fluviométricas utilizados na pesquisa como código e nome das estações na rede hidrometorológica nacional, rio onde está instalada a seção de monitoramento de vazão, número de anos da série histórica sem falhas $(\mathrm{N})$, período de medição $(\mathrm{P})$, coordenadas geográficas e área de drenagem $\left(\mathrm{A}_{\mathrm{D}}\right)$.

Tabela 1. Código e localização das estações fluviométricas da bacia do rio Juruena utilizadas neste estudo.

\begin{tabular}{|c|c|c|c|c|c|c|c|c|c|}
\hline ID & Código & Nome & Rio & Cidade & $\mathbf{N}$ & $\mathbf{P}$ & Latitude & Longitude & $\mathbf{A}_{\mathrm{D}}$ \\
\hline 1 & 17130000 & Foz do Juruena & Juruena & Apiacás & 6 & 1986-1996 & $-09^{\circ} 55^{\prime} 00^{\prime \prime}$ & $-58^{\circ} 17^{\prime} 00^{\prime \prime}$ & $182,458.3$ \\
\hline 2 & 17093000 & Fontanilhas & Juruena & Juína & 30 & $1978-2013$ & $-11^{\circ} 21^{\prime} 30^{\prime \prime}$ & $-58^{\circ} 20^{\prime} 34^{\prime \prime}$ & $55,924.2$ \\
\hline 3 & 17123000 & Rio Arínos & Arínos & Juara & 9 & $2000-2013$ & $-10^{\circ} 38^{\prime} 21^{\prime \prime}$ & $-58^{\circ} 00^{\prime} 15^{\prime \prime}$ & $57,110.1$ \\
\hline 4 & 17120000 & Porto dos Gaúchos & Arínos & Porto dos Gaúchos & 25 & $1973-2007$ & $-11^{\circ} 32^{\prime} 12^{\prime \prime}$ & $-57^{\circ} 25^{\prime} 25^{\prime \prime}$ & $37,069.6$ \\
\hline 5 & 17095000 & Fazenda Tombador & Rio do Sangue & Brasnorte & 21 & $1984-2013$ & $-11^{\circ} 43^{\prime} 05^{\prime \prime}$ & $-58^{\circ} 02^{\prime} 53^{\prime \prime}$ & $24,723.1$ \\
\hline 6 & 17122000 & Rio dos Peixes & Rio dos Peixes & Juara & 6 & $2000-2007$ & $-10^{\circ} 82^{\prime} 00^{\prime \prime}$ & $-57^{\circ} 72^{\prime} 00^{\prime \prime}$ & $14,410.5$ \\
\hline 7 & 17091000 & Fazenda Tucunaré & Juruena & Sapezal & 9 & $1993-2013$ & $-13^{\circ} 27^{\prime} 39^{\prime \prime}$ & $-59^{\circ} 00^{\prime} 28^{\prime \prime}$ & $4,357.9$ \\
\hline
\end{tabular}

ID: identificação na Figura 1; N: número de anos sem falhas; P período com dados consistidos; e $A_{D}$ : área de drenagem $(\mathrm{km})^{2}$. Fonte: Matos et al. (2020)

\section{Cálculo do Volume do Reservatório}

Uma equação de regularização é uma função como apresentada na Equação 1.

$$
R(t)=\frac{Q_{r}(t)}{\bar{Q}}
$$

em que: $\mathrm{Q}_{\mathrm{r}}(\mathrm{t})$ é a vazão regularizada (demandada) em função do tempo $\mathrm{t} \mathrm{em} \mathrm{m}^{3} \mathrm{~s}^{-1} ; \overline{\mathrm{Q}}$ é a vazão média de longa duração do curso de água $\mathrm{em}^{3} \mathrm{~s}^{-1}$. Destaca-se que a vazão média de longa duração $(\bar{Q})$ foi regionalizada para a bacia do rio Juruena e as equações podem ser obtidas no trabalho de Matos et al. (2020).

A capacidade mínima do reservatório $(\mathrm{Cr})$ para atender a Equação 1 (Equação de regularização) foi obtida pela diferença entre o volume que seria necessário para atender a vazão requerida no período mais crítico e o volume que aflui ao reservatório no mesmo período. Esse volume do reservatório foi obtido calculando a diferença das vazões na escala diária e para diferentes valores de R(t) (Equação 1) que representa a parcela da vazão média de longa duração que será regularizada. $\mathrm{O}$ valor de $\mathrm{Cr}$ foi calculado para vários períodos de estiagem e o valor mais crítico foi considerado.

Após a obtenção do volume do reservatório para diferentes $\mathrm{R}(\mathrm{t})$ plotou-se a curva de

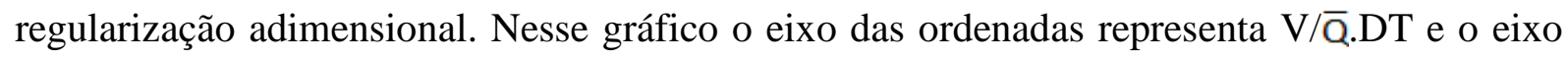


das abscissas $\mathrm{Q}_{\mathrm{r}} / \overline{\mathrm{Q}}$ em que $\mathrm{V}$ representa o volume do reservatório, $\overline{\mathrm{Q}}$ a vazão média de longa duração, $\mathrm{Q}_{\mathrm{r}}$ a vazão regularizada e DT o intervalo de tempo de medição da vazão. A evaporação do lago não foi considerada nesse cálculo.

\section{Regionalização da Curva de Regularização}

Para regionalização, as curvas de regularização de todas as estações fluviométricas foram plotadas em um único gráfico. Após esse procedimento foi ajustado aos dados um modelo do tipo potencial como apresentado na Equação 2.

$$
\mathrm{Y}=\mathrm{a}(\mathrm{X})^{\mathrm{b}}
$$

em que: Y representa (V/Q.DT); X representa $\mathrm{Q}_{\mathrm{r}} / \overline{\mathrm{Q}}$; e a e b são coeficientes da equação que foram obtidos por meio de regressão não linear.

A qualidade do ajuste da Equação 2 a série de dados foi realizada por meio do coeficiente de determinação $\left(\mathrm{R}^{2}\right)$ e do índice de eficiência de Nash-Sutcliffe ( $\left.\mathrm{E}_{\mathrm{NS}}\right)$ apresentado na Equação 3.

$$
\mathrm{ENS}_{\mathrm{NS}}=1-\left[\frac{\sum_{\mathrm{i}=1}^{\mathrm{N}}(\mathrm{Oi}-\mathrm{Pi})^{2}}{\sum_{\mathrm{i}=1}^{\mathrm{N}}(\mathrm{Oi}-\mathrm{O})^{2}}\right]
$$

em que: $\mathrm{P}_{\mathrm{i}}$ é o valor estimado com o modelo - Equação 2; $\mathrm{O}_{\mathrm{i}}$ é o valor observado; $\mathrm{O}$ é a média dos valores observados e $\mathrm{N}$ é o número de valores da amostra.

Mais detalhes sobre a metodologia de cálculo do volume do reservatório e da regionalização da curva de regularização podem ser obtidos em Tucci e Clarke (2016) e Tucci (2002). Estes autores abordam as principais metodologias existentes para regionalização hidrológica e aplicações em diferentes estudos técnicos na área de engenharia de recursos hídricos.

\section{Resultados e Discussão}

Na Figura 2 estão apresentadas as curvas adimensionais de regularização de vazão das sete seções de monitoramento de vazão da bacia do rio Juruena. Percebe-se que as curvas das seções 17123000 e 1713000 possuem comportamento próximo. 
Research, Society and Development, v. 9, n. 10, e2539108637, 2020

(CC BY 4.0) | ISSN 2525-3409 | DOI: http://dx.doi.org/10.33448/rsd-v9i10.8637

$\mathrm{Na}$ Figura 2, fica evidente, para todas as estações, que se for preciso uma regularização de até $25 \%$ da vazão média de longa duração não há necessidade de construir reservatório. Para valores de regularização superiores a $25 \%$ existe uma distinção de comportamento para as diferentes regiões da bacia.

O estabelecimento dessa curva para diferentes pontos da bacia subsidia os estudos hidrolólogicos de barramentos para irrigação e geração de energia elétrica.

Figura 2. Curva de regularização de vazões das seções de monitoramento da bacia do rio Juruena apresentadas na Tabela 1.

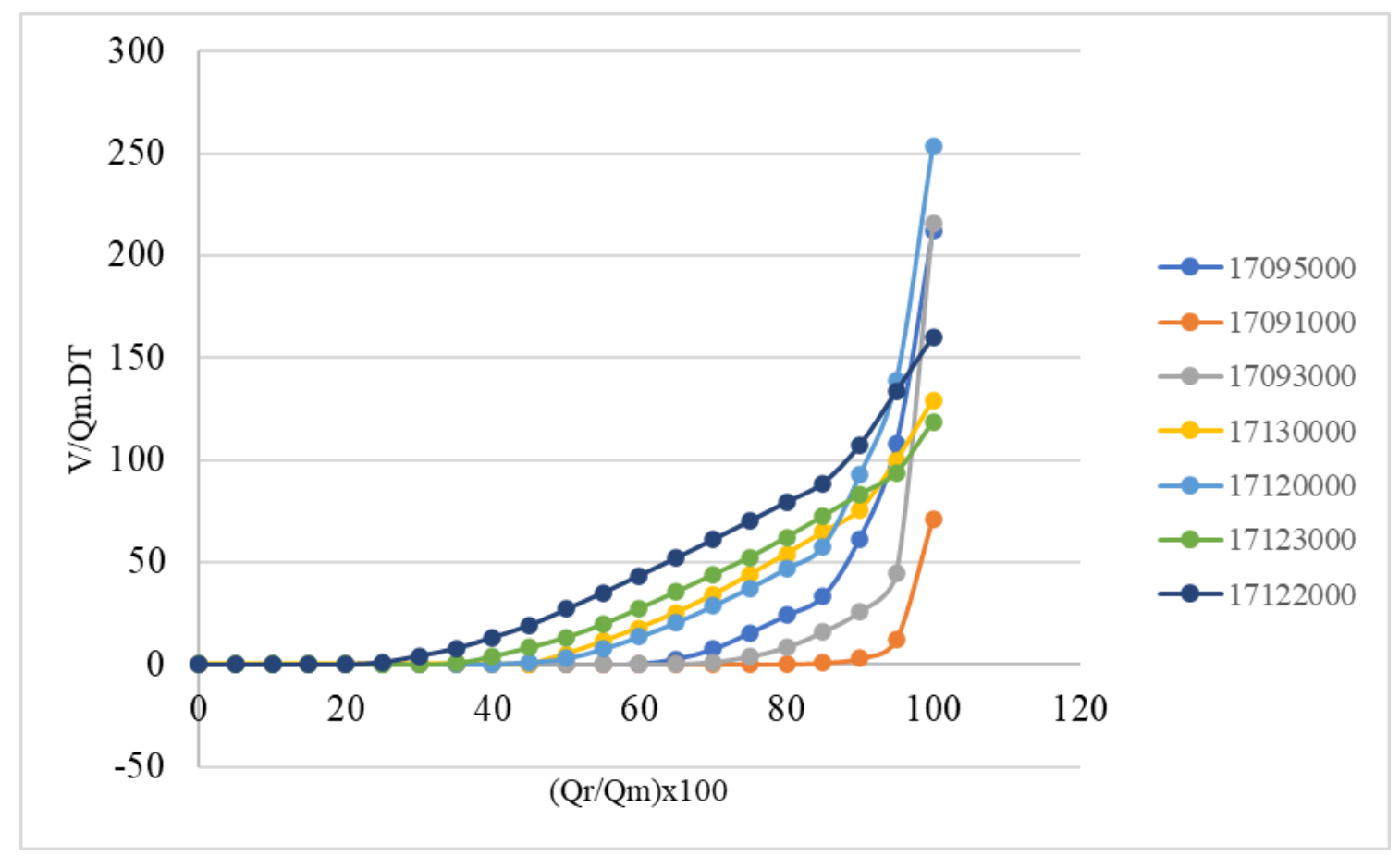

Fonte: Os autores.

$\mathrm{Na}$ Tabela 2 estão apresentados os ajustes estatísticos das curvas de regularização conforme a Equação 2. Para a estação 17091000 (Fazenda Tucunaré) não foi realizado o ajuste, pois nesta seção para regularização de até $80 \%$ da vazão média não é necessário construir reservatório (Figura 2).

Segundo Tucci (2002) para regionalização da curva de regularização deve-se estabelecer um intervalo apropriado para a porcentagem de vazão média a ser regularizada [(Qr/Qm)x100], visto que no extremo superior existe alta variação de tendência da curva devido à magnitude das vazões envolvidas e do volume necessário para regularizá-las. Ainda de acordo com esse autor dificilmente procura-se regularizar $100 \%$ da vazão média de um rio. Nesse contexto, foi realizado o ajuste das curvas de regularização (Figura 2) para intervalo de 
(Qr/Qm)x100 entre 0 e 90\%, com exceção para a estação fluviométrica 17091000. Nesta estação a regularização é necessária quando (Qr/Qm)x100 supera 80\% inviabilizando o ajuste da curva.

Tabela 2. Ajuste estatístico das curvas de regularização conforme a Equação 2 .

\begin{tabular}{ccccc}
\hline ID & Código & Equação & $\mathbf{R}^{\mathbf{2}}$ & $\mathbf{E}_{\mathbf{N S}}$ \\
\hline 1 & 17130000 & $\mathrm{Y}=4,429 \mathrm{E}-06 .(\mathrm{X})^{3,7119}$ & 0,98 & 0,98 \\
2 & 17093000 & $\mathrm{Y}=5,798 \mathrm{E}-18 \cdot(\mathrm{X})^{9,5445}$ & 0,99 & 0,99 \\
3 & 17123000 & $\mathrm{Y}=1,344 \mathrm{E}-04 .(\mathrm{X})^{2,9719}$ & 0,99 & 0,99 \\
4 & 17120000 & $\mathrm{Y}=2,044 \mathrm{E}-08 .(\mathrm{X})^{4,9284}$ & 0,98 & 0,98 \\
5 & 17095000 & $\mathrm{Y}=1,547 \mathrm{E}-15 \cdot(\mathrm{X})^{8,4898}$ & 0,99 & 0,99 \\
6 & 17122000 & $\mathrm{Y}=2,401 \mathrm{E}-03 \cdot(\mathrm{X})^{2,3778}$ & 0,99 & 0,99 \\
\hline
\end{tabular}

Fonte: Os autores.

Devido a pequena quantidade de estações com série histórica na bacia não foi possível buscar uma tendência comum entre as curvas. Assim foi realizado o ajuste da Equação 2 para cada seção de monitoramento. Percebe-se na Tabela 2 que a qualidade do ajuste foi muito bom. Todos os valores de coeficiente de determinação e $E_{N S}$ ficaram superior a 0,98 o que indica precisão e acurácia da Equação.

A baixa quantidade de estações na bacia, bem como séries históricas com menos de 30 anos de dados aumentam as incertezas dos resultados apresentados nesse estudo. Por isso, devem ser utilizados com um maior cuidado por técnicos que irão executar dimensionamento de reservatórios na bacia hidrográfica. Fica evidente a necessidade de aumentar a rede de monitoramento fluviométrico na bacia, principalmente, em cursos de água menores para embasamento do planejamento e gestão dos recursos hídricos.

Sabe-se que existe recomendação em relação à utilização de séries históricas de 30 anos de dados nas análises hidrológicas, entretanto, na maior parte dos estudos realizados no Brasil não se consegue contemplar esse período de informações em função das limitações da base de dados. Na região amazônica, onde a bacia do Juruena está inserida a densidade de estações é muito baixa comparativamente a outras regiões do país. Algumas localidades são de difícil acesso o que resulta em falta de manutenção dos equipamentos e consequentemente em falhas no registro. A estação Foz do Juruena, por exemplo, está localizada dentro de um parque nacional cuja a floresta está totalmente preservada. Neste tipo de situação o acesso ocorre por barco ou transporte aéreo. Tudo isso contribui para a existência de falhas nos registros e série históricas de curta duração, gerando incertezas nas análises. 
Mesmo sabendo que o ideal seria ter uma base de dados maior não se pode deixar de obter informações importantes, como as deste trabalho, esperando até o momento em que haverá séries históricas perfeitas para a realização dos estudos hidrológicos. É preciso ter informações para tomada de decisão na bacia, pois obras hidráulicas já estão sendo planejadas e executadas na bacia.

\section{Considerações Finais}

A regionalização das curvas de regularização de vazão da bacia do rio Juruena foi realizada. As equações de regionalização propostas possuem precisão e acurácia e poderão embasar projetos de regularização de vazão para irrigação, abastecimento público e geração de energia elétrica.

O trabalho evidencia a necessidade de aumentar a rede de monitoramento fluviométrico na bacia do rio Juruena, principalmente em cursos de água menores para embasamento do planejamento e gestão dos recursos hídricos. Nesse contexto, com novos postos de medições de vazão bem como extensão das séries históricas existentes será possível atualizar as equações de regionalização ajustadas neste estudo e reduzir as incertezas relacionadas a regularização de vazão na bacia.

\section{Referências}

Collischonn, W., \& Dornelles, F. (2015). Hidrologia: para engenharia e ciências ambientais. Porto Alegre: ABRH. 336 p.

Lopes, T. R., Prado, G., Zolin, C. A., Paulino, J., \& Antoniel, L. S. (2016). Regionalização de Vazões Máximas e Mínimas para a Bacia do Rio Ivaí, PR. Irriga, 21(1), 188-201.

Matos, T. S., Uliana, E. M., Martins, C. A. S., \& Rapalo, L. M. C. (2020). Regionalização de vazões máximas, mínima e média para a bacia hidrográfica do Rio Juruena, Brasil. Revista Ambiente \& Água, 15(3), e2418.

Pruski, F. F., Silva, D. D., \& Koetz, M. (2006). Estudo de vazão em cursos d'água. Viçosa: AEAGRI. $151 \mathrm{p}$. 
Tucci, C. E. M. (2002). Regionalização de Vazões. Porto Alegre: Editora da Universidade/UFRGS. 256p.

Tucci, C. E. M., \& Clarke, R. T. (2016). Regionalização Hidrológica. In: Paiva, J. B. D., \& Paiva, E. M. C. D. Hidrologia Aplicada à Gestão de Pequenas Bacias Hidrográficas (pp. 169-222). Porto Alegre: ABRH.

Uliana, E. M., Souza, L. G. S., Silva, D. D., Souza, A. P., Almeida, F. T., \& ARAUJO, H. B. (2016). Regionalização de vazões para o médio e alto rio Teles Pires - MT. Revista de Ciências Agrárias, 59(4), 333-338.

\section{Porcentagem de contribuição de cada autor no manuscrito}

João Paulo de Amorim Oliveira - 35\%

Eduardo Morgan Uliana - 25\%

Múcio André dos Santos Alves Mendes - 20\%

Luana Lisboa - $20 \%$ 